\title{
Inversion of Marine Radar Images for Surface Wave Analysis
}

\author{
José C. NiETo Borge \\ German Aerospace Center Oberpfaffenhofen, Wessling, Germany \\ GERMÁN RODRÍGUEZ RODRÍGUEZ \\ Universidad de Las Palmas de Gran Canaria, Las Palmas, Canary Islands, Spain \\ KATRIN HESSNER \\ Ocean Waves GmbH, Munstermannskamp, Jüneburg, Germany \\ PALOMA IZQuiERdo GonZÁLEZ \\ Instituto Superior Técnico, Lisbon, Portugal
}

(Manuscript received 14 August 2002, in final form 27 October 2003)

ABSTRACT

\begin{abstract}
A method to estimate sea surface elevation maps from marine radar image sequences is presented. This method is the extension of an existing inverse modeling technique to derive wave spectra from marine radar images, which assumes linear wave theory with temporal stationarity and spatial homogeneity of the observed sea surface elevation. The proposed technique to estimate wave elevation maps takes into account a modulation transfer function (MTF), which describes the radar imaging mechanisms at grazing incidence and horizontal polarization. This MTF is investigated and empirically determined by wave measurements and numerical simulations. The numerical simulations show that shadowing is the dominant effect in the radar imaging mechanism at grazing incidence and horizontal polarization. Further comparisons of wave spectra, as well as comparisons of the wave height probability distributions obtained by the wave elevation maps and the corresponding buoy measurements with the theoretical Rayleigh distribution, confirm the applicability of the proposed method.
\end{abstract}

\section{Introduction}

It is known that under various conditions signatures of the sea surface are visible in the near range $(<3 \mathrm{n}$ $\mathrm{mi})$ of marine radar images. These signatures are known as sea clutter because they are undesirable for navigation purposes and generally suppressed by filter algorithms. Sea clutter is mainly due to the backscatter through Bragg resonance with ocean waves of wavelengths similar to that of the transmitted electromagnetic waves. The longer waves become visible in the radar images because they modulate the sea clutter signals. The modulation is a nonlinear process mainly affected by three effects: hydrodynamic modulation (HM), tilt modulation (TM), and shadowing ( $\mathrm{SH})$, where HM describes the modulation of the energy of the ripples by the interaction with the longer waves, TM the modulation due to the changes of the effective incidence angle along

Corresponding author address: Dr. José C. Nieto Borge, German Aerospace Center (DLR), Remote Sensing Technology Institute (IMF), Oberpfaffenhofen, D-82234 Wessling, Germany. E-mail: jose.c.nieto@dlr.de the long wave slope, and SH the partially shadowing of the sea surface by higher waves (Lee et al. 1995; Plant and Keller 1990; Wenzel 1990). Hence the sea clutter radar image intensities do not map on a one-to-one scale the ocean surface elevation. Since marine radar systems give the opportunity to scan the sea surface with high temporal and spatial resolution (see Fig. 1), it allows monitoring of the sea state (Young et al. 1985; Ziemer and Rosenthal 1991; Nieto Borge et al. 1999b; Nieto Borge and Guedes Soares 2000; Hessner et al. 2001). The objective of the present paper is to demonstrate the ability to invert sea clutter images into sea surface elevation maps, which are estimations of the waves imaged by the radar. This inversion scheme allows the investigation of single wave properties, such as, for example, wave height and crest steepness. This is especially of importance for the investigation of rogue waves (singular appearing wave phenomenon that cannot be described by common wave statistics). The rest of the paper is organized as follows. Section 2 gives a short review of the inverse modeling technique for the determination of directional wave spectra from marine ra- 


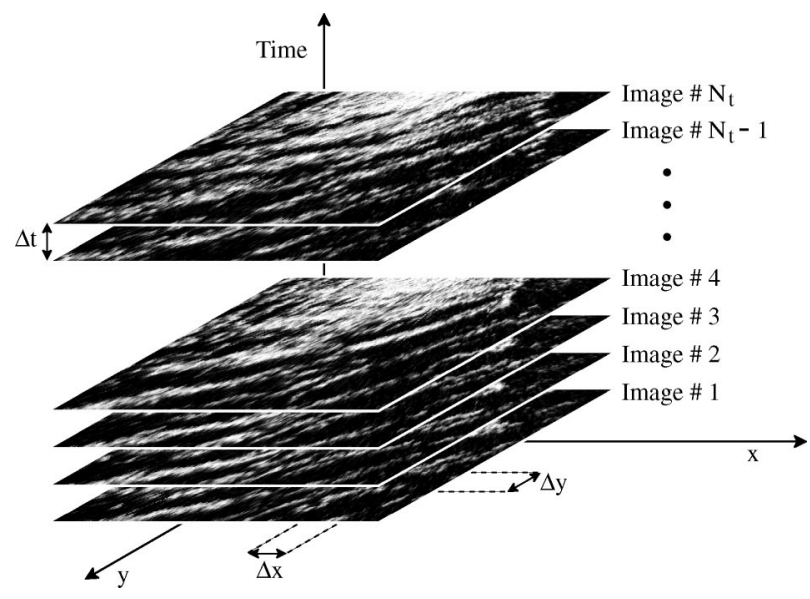

FIG. 1. Temporal sequence of sea clutter images. The grayscale corresponds to the radar backscatter strength. The temporal resolution $\Delta t$ is given by the antenna rotation period. The spatial resolutions $\Delta x$ and $\Delta y$ are dependent on the azimuthal resolution due to the effective aperture of the antenna and on the range resolution due to the radar pulse length.

dar imagery. Section 3 discusses the empirical modulation transfer function (MTF) that has been applied. Here results from experimental data and theoretical investigations by means of numerical simulations are presented. In section 4 the scheme of the inversion technique is given. In section 5 a method for the estimation of wave height probability distribution from temporal sequences of 2D wave fields is presented. Summary and conclusions are given in section 6 .

\section{Wave spectrum estimation}

To derive sea state parameters from sea clutter time series $g(\mathbf{r}, t)$ the concept of sea state is taken into account, which is based on linear wave theory and assumes spatial homogeneity and temporal stationarity of the wave field. Under these assumptions, sea states are described as a Gaussian zero-mean random processes varying in space $\mathbf{r}=(x, y)$ and time $t$, with vertical wave elevations over the mean sea level $\eta$ having the following spectral representation:

$$
\left.\eta(\mathbf{r}, t)=\int_{\Omega_{\mathbf{k}, \omega}} \exp [i \mathbf{k} \cdot \mathbf{r}-\omega t)\right] d Z(\mathbf{k}, \omega),
$$

where $\mathbf{k}=\left(k_{x}, k_{y}\right)$ is the wavenumber vector, $\omega$ is the angular frequency, $d Z(\mathbf{k}, \omega)$ is the random complex amplitude, and the integration domain $\Omega_{\mathbf{k}, \omega}$ is defined as the admissible range of $\mathbf{k s}$ and $\omega$ s for swell and wind sea states. Hence the $3 \mathrm{D}$ wave spectrum is defined as $\chi(\mathbf{k}, \omega) d^{2} \mathbf{k} d \omega=\varepsilon\left[d Z(\mathbf{k}, \omega) d Z^{*}(\mathbf{k}, \omega)\right]$, where $\varepsilon$ is the expectation operator, and the superscript $(*)$ indicates the complex conjugate. Assuming the linear wave theory, ocean surface waves are dispersive with a dispersion relation given by

$$
\omega=\varpi(\mathbf{k})=\sqrt{g k \tanh (k h)}+\mathbf{k} \cdot \mathbf{U},
$$

where $k=\|\mathbf{k}\|, g$ is the acceleration due to gravity, $h$ is the water depth, and $\mathbf{U}=\left(U_{x}, U_{y}\right)$ is the surface current.

The Fourier decomposition of sea clutter time series $g(\mathbf{r}, t)$ provides 3D image spectra [denoted here as $\gamma(\mathbf{k}$, $\omega)$, rather than $3 \mathrm{D}$ wave spectra $\chi(\mathbf{k}, \omega)$. To estimate $\chi(\mathbf{k}, \omega)$ from the image spectrum, an inverse modeling technique has to be applied to select the wave spectral components within $\gamma(\mathbf{k}, \omega)$ (Hessner et al. 1999). The basic steps to estimate the wave spectra are as follows.

1) The current is derived by determining the deviation between the measured dispersion relation and the theoretical dispersion relation for linear surface waves (Young et al. 1985). Here an improved method has been applied, which also takes into account aliased wave signals and signals due to higher harmonics (Senet et al. 2001). This improvement allows the system to be used on board moving ships. In this case, the determined current is due to the relative motion between the radar (ship) and the wave field. The surface current is then the deviation between relative motion and ship speed. Note that for the determination of the wave spectrum, only the relative motion is relevant.

2) The wave spectral components are filtered. Taking into account the dispersion relation for linear gravity waves [Eq. (2)], the 2D filtered image spectrum is estimated as (Young et al. 1985)

$$
\Psi_{r}(\mathbf{k})=2 \int_{\omega>0} \gamma(\mathbf{k}, \omega) \delta[\omega-\varpi(\mathbf{k})] d \omega,
$$

where $\delta\left(\alpha-\alpha_{0}\right)$ is the Dirac's delta. In the discrete space the width of the filter depends on the frequency and wavenumber resolutions, which are related to the length of the image time series and the size of the analysis area, respectively. Note that in the following when we refer to the image spectrum, $\Psi_{r}(\mathbf{k})$ is considered.

3) Significant wave height is estimated. In contrast to in situ wave measurements (e.g., buoys), the significant wave height $H_{s}$ cannot be in fact directly determined from marine sea clutter images. The first method to obtain wave height information from radar imagery was proposed by Alpers and Hasselmann (1982). For SAR imagery, they proposed that the significant wave height is proportional to the signalto-noise ratio (SNR) of the radar backscatter. This method was validated by Plant and Zurk (1997). This idea was first applied to marine radar image sequences by Ziemer and Günther (1994). In contrast to the application for SAR imagery, the SNR for marine radar images were obtained from the $3 \mathrm{D}$ image spectrum by means of a dispersion relation. Since the first application of this empirical correlation, the definition of SNR has been improved (Nieto Borge 1998). The capability of this method has been demonstrated in several field experiments (Nieto 
Borge et al. 1999a; Hessner et al. 2001). For grazing incidence, there is an alternative method to estimate $H_{s}$ based on shadowing statistics proposed by Wenzel (1990). This method was validated by Henschel et al. (1995) and Buckley and Aler (1997, 1998).

\section{Modulation transfer function}

For horizontal polarization and at grazing incidence, a difference between the image spectra from marine radar imagery and the corresponding spectra from in situ sensors can be observed. This difference is due to the radar wave imaging mechanisms like, for example, shadowing and/or tilt modulation (Nieto Borge 1997; Seemann et al. 1997). By using an MTF, this difference can be minimized (Plant 1988; Ziemer and Günther 1994).

Figure 2a shows an example of a normalized wave spectrum from a buoy record (thin solid line) and the corresponding normalized spectrum inferred from the radar measurements. The dotted line refers to the spectrum derived without an MTF (here called image spectrum) and the thick solid line refers to the spectrum derived with an MTF (here called wave spectrum). A significant difference of the normalized energy can be seen especially for high wavenumbers. The empirical determination of the MTF is described in the following section.

\section{a. Modulation transfer function derived from in situ measurements}

The empirical modulation transfer function can be determined by

$$
|\mathcal{M}(k)|^{2}=F_{r}(k) / F_{\text {is }}(k),
$$

where $F_{r}(k)$ is the $1 \mathrm{D}$ wavenumber spectrum derived from the radar measurements:

$$
F_{r}(k)=\int_{-\pi}^{\pi} \Psi_{r}[\mathbf{k}(k, \theta)] k d \theta,
$$

and $F_{\text {is }}(k)$ is the corresponding spectrum derived from the frequency spectrum $S(\omega)$ obtained from heave time series measured by in situ sensors:

$$
F_{\text {is }}(k)=S[\omega(k)] \frac{d \omega}{d k} .
$$

Equation (6) is valid for those cases where the Doppler shift effect in frequency due to a current is negligible.

To determine the $|\mathcal{M}(k)|^{2}$ shape, a measuring campaign was carried out in the Bay of Biscay, in a deep water area $(h \approx 600 \mathrm{~m})$ close to the Spanish northern coast. Two different measuring devices were used: a pitch-roll WaveScan buoy as the reference in situ sensor, and a WaMos II (Wave Monitoring System), an operational commercial system developed specifically to measure sea states with standard marine radars (Dittmer
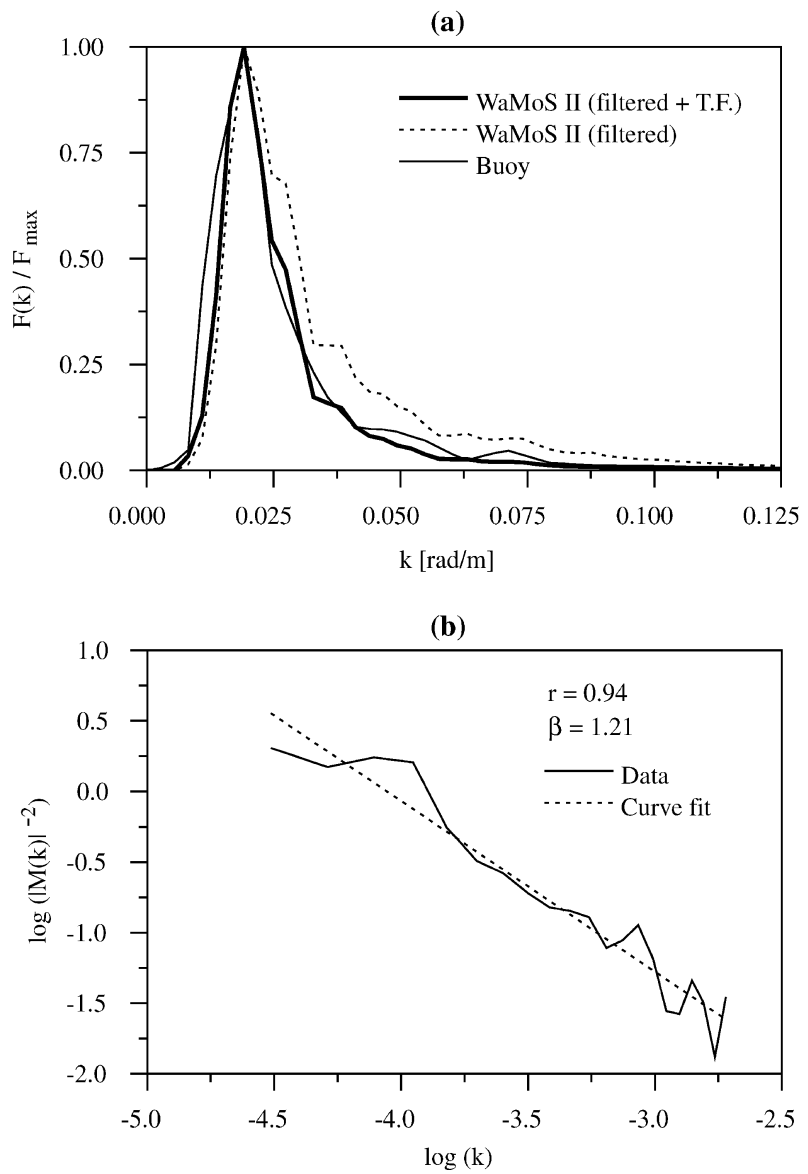

FIG. 2. (a) The wavenumber spectrum as obtained by a WaveScan buoy (thin solid line) and radar-based system WaMoS II in the Bay of Biscay. The dotted line indicates the radar image spectrum, and the thick solid line represents the corresponding wave spectrum using the empirical modulation transfer function. (b) The fit of the modulation transfer function exponent $\beta$. WaMoS II data were acquired by the marine radar on board the maritime rescue vessel Golfo de Vizcaya at the Bay of Biscay, close to the northern coast of Spain at 1508 UTC 14 Feb 1995.

1995). Analyzing the measurements, a power decay law for the transfer function was determined, $|\mathcal{M}(k)|^{2} \propto k^{\beta}$, giving a mean value of the exponent $\beta$ was greater than 1 in most of the cases (see Fig. $2 \mathrm{~b}$ and Table 1), presenting a mean value $\bar{\beta}=1.2$. To obtain more information about $\mid \mathcal{M k})\left.\right|^{2}$, numerical simulations of sea clutter time series have been carried out.

\section{b. Modulation transfer function estimation from numerical simulations}

\section{1) Numerical Simulation of SEA CLUTTER IMAGES}

To derive some of the marine radar imaging properties a numerical stochastic simulation model of the sea surface has been used. Assuming the linear wave model, and considering a known directional wave spectrum 
TABLE 1. Fitted values for the transfer function exponent $\beta$. The obtained mean value is $\bar{\beta}=1.2$. In addition, the table contains the estimated values of the significant wave height $H_{s}$ and peak period $T_{p}$ from the buoy records (WaveScan) and from the marine radar (WaMoS II). The data were measured in the Bay of Biscay close to the Spanish northern coast in deep waters $(h \approx 600 \mathrm{~m})$. The WaMoS II system was connected to the navigation radar on board the maritime rescue vessel Golfo de Vizcaya.

\begin{tabular}{|c|c|c|c|c|c|}
\hline Data and time (UTC) & $H_{s}(\mathrm{~m})($ WaveScan $)$ & $H_{s}(\mathrm{~m})(\mathrm{WaMoS} \mathrm{II})$ & $T_{p}(\mathrm{~s})($ WaveScan $)$ & $T_{p}(\mathrm{~s})(\mathrm{WaMoS} \mathrm{II})$ & $\beta$ \\
\hline 1155 UTC 9 Feb 1995 & 2.3 & 2.4 & 10.8 & 11.8 & 1.21 \\
\hline 1158 UTC 9 Feb 1995 & 2.3 & 2.5 & 10.8 & 12.9 & 1.01 \\
\hline 1200 UTC 9 Feb 1995 & 2.3 & 2.2 & 10.8 & 09.6 & 1.25 \\
\hline 1203 UTC 9 Feb 1995 & 2.3 & 2.7 & 10.8 & 10.8 & 1.32 \\
\hline 1206 UTC 9 Feb 1995 & 2.3 & 2.8 & 10.8 & 11.9 & 1.21 \\
\hline 1500 UTC 9 Feb 1995 & 1.9 & 2.2 & 10.4 & 11.5 & 1.28 \\
\hline 1502 UTC 9 Feb 1995 & 1.9 & 1.8 & 10.4 & 12.1 & 1.25 \\
\hline 1505 UTC 9 Feb 1995 & 1.9 & 1.7 & 10.4 & 11.7 & 1.11 \\
\hline 1508 UTC 9 Feb 1995 & 1.9 & 2.3 & 10.4 & 11.5 & 1.28 \\
\hline 1511 UTC 9 Feb 1995 & 1.9 & 2.2 & 10.4 & 12.1 & 1.17 \\
\hline 1514 UTC 9 Feb 1995 & 1.9 & 2.1 & 10.4 & 09.6 & 1.19 \\
\hline 1200 UTC 13 Feb 1995 & 4.7 & 5.1 & 16.5 & 16.0 & 0.99 \\
\hline 1202 UTC 13 Feb 1995 & 4.7 & 4.8 & 16.5 & 14.7 & 1.01 \\
\hline 1205 UTC 13 Feb 1995 & 4.7 & 4.6 & 16.5 & 13.7 & 1.25 \\
\hline 1208 UTC 13 Feb 1995 & 4.7 & 4.9 & 16.5 & 13.9 & 1.37 \\
\hline 1211 UTC 13 Feb 1995 & 4.7 & 4.6 & 16.5 & 15.5 & 1.22 \\
\hline 1214 UTC 13 Feb 1995 & 4.7 & 4.5 & 16.5 & 16.6 & 1.24 \\
\hline 1500 UTC 14 Feb 1995 & 4.3 & 4.0 & 14.4 & 14.3 & 1.04 \\
\hline 1502 UTC 14 Feb 1995 & 4.3 & 4.1 & 14.4 & 15.0 & 1.22 \\
\hline 1505 UTC 14 Feb 1995 & 4.3 & 4.5 & 14.4 & 15.2 & 0.97 \\
\hline 1508 UTC 14 Feb 1995 & 4.3 & 4.4 & 14.4 & 14.2 & 1.21 \\
\hline 1511 UTC 14 Feb 1995 & 4.3 & 4.3 & 14.4 & 14.7 & 1.23 \\
\hline 1514 UTC 14 Feb 1995 & 4.3 & 3.8 & 14.4 & 15.0 & 1.08 \\
\hline
\end{tabular}

$\Psi(\mathbf{k})$, the simulated wave elevation $\eta(\mathbf{r}, t)$ is calculated as (Nieto Borge 1997)

$$
\eta(\mathbf{r}, t)=\sum_{m=1}^{N_{x}} \sum_{n=1}^{N_{y}} \hat{A}_{m n} \cos \left[\mathbf{k}_{m n} \cdot \mathbf{r}-\varpi\left(\mathbf{k}_{m n}\right) t-\varphi_{m n}\right],
$$

where $\varphi_{m n}$ are uniformly distributed random phases on the interval $[-\pi, \pi)$ and the amplitudes are given by $\hat{A}_{m n}=\sqrt{2 \hat{\Psi}\left(\mathbf{k}_{m n}\right) \Delta k_{x_{m}} \Delta k_{y_{n}}}$, being $[\nu \hat{\Psi}(\mathbf{k}) / \Psi(\mathbf{k})] \mathrm{a} \chi_{\nu}^{2}$-distributed random variable. This work used $\nu=2$ for the number of degrees of freedom.

For far-range and horizontal polarization, geometrical optics approximation is assumed (Plant and Keller 1990). Hence, for grazing incidence the local incidence

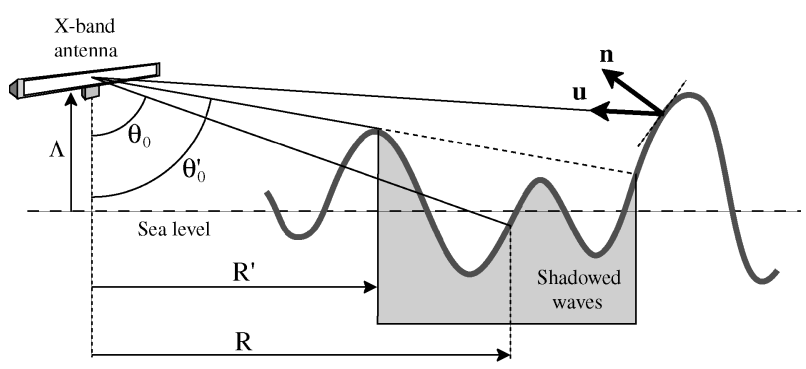

FIG. 3. Scheme showing the geometrical method to simulate wave shadowing. A wave facet, at range $R$, is shadowed to the antenna if there is another facet in the same azimuthal angle with $R^{\prime}<R$, for which $\theta_{0}^{\prime}<\theta_{0}$. In addition, the figure shows the mechanism to simulate tilt modulation at grazing incidence by using the scalar product of the 3D unit wave surface exterior vector $\mathbf{n}$ and the $3 \mathrm{D}$ unit vector from the sea surface to the antenna $\mathbf{u}$. angle on an illuminated facet (which is related to the radar resolution cell located at point $\mathbf{r}$ and at time $t$ ) is $\theta_{0}(\mathbf{r}, t)=\tan ^{-1}\{R(\mathbf{r}) /[\Lambda-\eta(\mathbf{r}, t)]\}$, where $R(\mathbf{r})$ is the range and $\Delta$ is the antenna height over the sea level (see Fig. 3). The facet will be shadowed to the antenna if another facet [located at the point $\mathbf{r}^{\prime}$, with $R^{\prime}=R\left(\mathbf{r}^{\prime}\right)$ $<R(\mathbf{r})$, at the same azimuthal angle than $\mathbf{r}$ ] has a local incidence angle $\theta_{0}^{\prime}=\theta_{0}\left(\mathbf{r}^{\prime} t\right) \geq \theta_{0}(\mathbf{r}, t)$. The simulated shadowed image is given by (Nieto Borge 1997)

$$
\sigma_{\mathrm{sh}}(\mathbf{r}, t)= \begin{cases}\sigma(\mathbf{r}, t), & \text { if no shadowing occurs } \\ 0, & \text { otherwise }\end{cases}
$$

where $\sigma(\mathbf{r}, t)$ is obtained by rescaling the values of $\eta(\mathbf{r}$, $t)$ into 256 gray levels ( 1 unsigned byte). Note that $\sigma(\mathbf{r}$, $t$ ) is only a first-order approximation of the digital radar output, as the radar intensity is not proportional to the wave height. Nevertheless, this is an adequate assumption for our investigation, as hydrodynamic and tilt modulation have a minor impact on the imaging mechanism compared to shadowing at grazing incidence (Lee et al. 1995; Seemann 1997).

At grazing incidence, the tilt modulation depends on each facet orientation and facet range (Nieto Borge 1997). This effect is simulated by using the scalar product between the 3D unit exterior normal vector $\mathbf{n}(\mathbf{r}, t)$ to the simulated surface, and the 3D unit vector from the facet to the antenna, $\mathbf{u}(\mathbf{r}, t)$ (see Fig. 3). Analytically, the normal exterior vector is given by $\mathbf{n}=\left(\boldsymbol{\rho}_{x} \times \boldsymbol{\rho}_{y}\right)$ $\left\|\boldsymbol{\rho}_{x} \times \boldsymbol{\rho}_{y}\right\|$, where $\boldsymbol{\rho}_{x}=(1,0, \partial \eta / \partial x)$ and $\boldsymbol{\rho}_{y}=(0,1$, $\partial \eta / \partial y)$ are the $3 \mathrm{D}$ tangent vectors on the surface 
(a) Simulated wave field

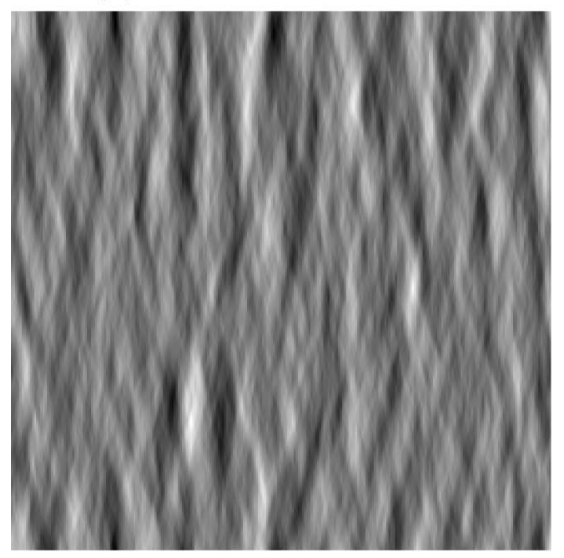

(c) Shadowed image

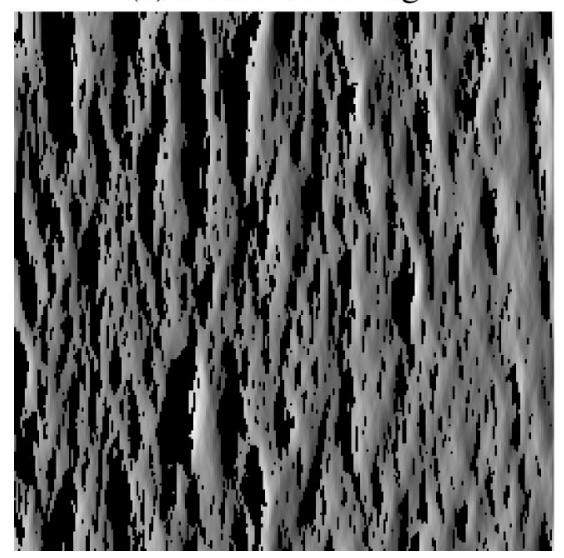

(b) Shadowing mask

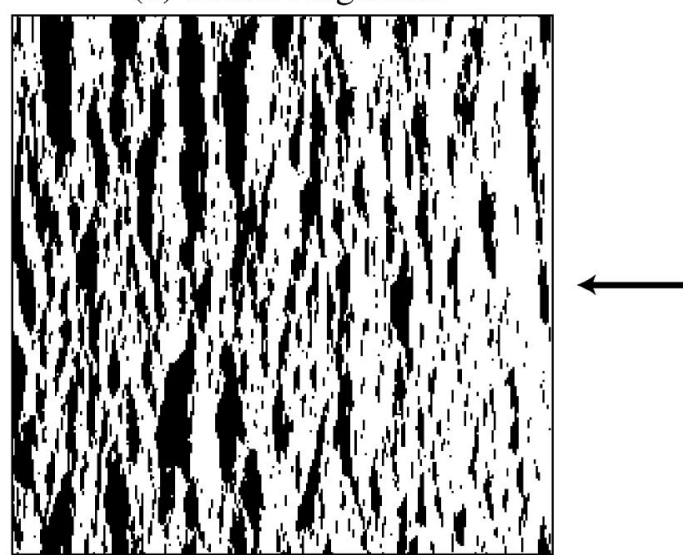

(d) Tilt modulated image

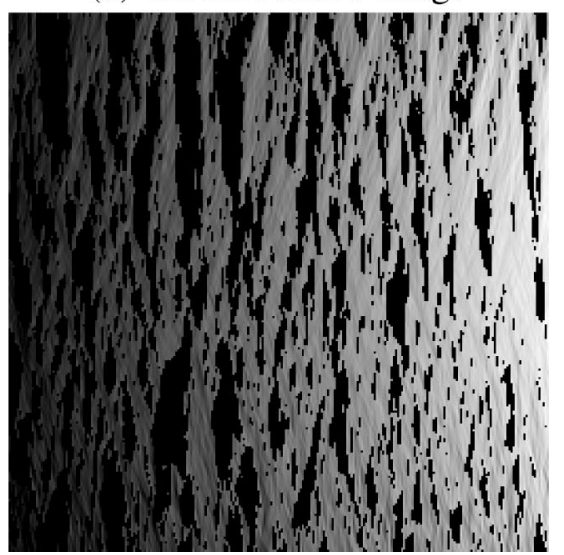

FIG. 4. Results of the sea state simulation of a rectangular area located $300 \mathrm{~m}$ off the antenna: (a) sea surface elevation where grayscale corresponds to $\eta$, (b) corresponding shadowing mask assuming an antenna $20 \mathrm{~m}$ above sea level, (c) simulated sea clutter image assuming shadowing and radar backscatter proportional to the surface elevation, and (d) simulated sea clutter image where additional tilt modulation has been applied. The arrow indicates the radar look direction.

$\eta(\mathbf{r}, t)$. Hence, the tilt simulated image is given by the factor $\mathcal{T}(\mathbf{r}, t)=\mathbf{n}(\mathbf{r}, t) \cdot \mathbf{u}(\mathbf{r}, t)$

$\sigma_{\text {tilt }}(\mathbf{r}, t)= \begin{cases}\mathcal{T}(\mathbf{r}, t), & \text { if } \mathcal{T}(\mathbf{r}, t)>0 \quad \text { and } \quad \sigma_{\text {sh }}(\mathbf{r}, t) \neq 0 \\ 0, & \text { otherwise. }\end{cases}$

Finally, the $\sigma_{\text {tilt }}(\mathbf{r}, t)$ values are coded with 256 gray levels. Figure 4 illustrates the results of a sea state simulation for a $2560 \mathrm{~m} \times 2560 \mathrm{~m}$ area. The simulation was carried out for a Wallops spectrum with $H_{s}=4 \mathrm{~m}$ and a peak frequency $f_{p}=0.08 \mathrm{~Hz}$. The upper-left panel shows the sea surface elevation where the light areas correspond to wave crest and the black areas to wave troughs. The upper-right panel shows the corresponding shadowing mask for an antenna at 300-m distance and $20 \mathrm{~m}$ above the mean sea level. Here black (white) indicates shadowed (illuminated) areas. The lower-left panel shows the combination of sea surface elevation and the shadowing mask, which represents the first- order approximation of the radar image. In the lowerright panel, the tilt modulation is also taken into account.

\section{2) MOdulation TRANSFER FUNCTION DERIVED FROM NUMERICAL SIMULATIONS}

To estimate the transfer function $|\mathcal{M}(k)|^{2}$, a set of numerical simulations has been carried out. Different sea state conditions were considered by applying different theoretical frequency spectra: Wallops (Huang 1981) for swell cases and JONSWAP for wind sea cases. For the directional spreading function $D(\omega, \theta)$, a squared cosine distribution was used:

$$
D(\omega, \theta)=\frac{2^{2 s-1}}{\pi} \frac{\Gamma^{2}(s+1)}{\Gamma(2 s+1)} \cos ^{2 s}\left(\frac{\theta-\bar{\theta}}{2}\right),
$$

where $\Gamma$ is the gamma function, and the mean wave direction is given by $\bar{\theta}$. The parameter $s$ leads the angular spreading; $s$ is a function of the wave frequency 
TABLE 2. Angular spreading $s_{\max }$ for the numerical sea state simulations of swell and wind sea.

\begin{tabular}{lcc}
\hline \hline Sea state & Peak frequencies $[\mathrm{Hz}]$ & $s_{\max }$ \\
\hline Swell & $0.08,0.09$ & 75 \\
& 0.1 & 25 \\
Wind sea & $0.1,0.12,0.14$ & 10 \\
\hline
\end{tabular}

$f=\omega / 2 \pi$. The parameterization for the function $s(f)$ given by Mitsuyasu et al. (1980) has been used,

$$
s(f)=s_{\max }\left(\frac{f}{f_{p}}\right)^{\mu},
$$

where $\mu$ has two different values depending on the wave frequency:

$$
\mu= \begin{cases}5, & \text { when } f \leq f_{p} \\ -2.5, & \text { when } f>f_{p} .\end{cases}
$$

For our simulations, three different values of significant wave height $(2,4$, and $6 \mathrm{~m})$ have been used for swell and wind sea cases. In addition, the angular spreading for the peak frequency was obtained taking into account empirical values of $s_{\max }$ determined for swell and wind sea (Goda 2000; Sorensen 1993). The used values of $s_{\max }$ are given in Table 2 .

For each sea state, 400 simulations with random phases and random amplitudes were carried out. For each simulation, $\beta$ was determined by taking shadowing only and shadowing plus tilt modulation into account. Mean and variance of $\beta$ for swell and wind sea are summarized in Table 3. Figure 5 shows the resulting power decay for $|\mathcal{M}(k)|^{2}$ for (Fig. 5a) a swell simulation and for (Fig. 5b) a wind sea simulation. Independent of the input wave spectra and the sea state parameters used for the simulations, $\beta$ was found in the range of 1.2 , which coincides with the result of the measurements presented before. The found values for $\beta$ do not differ significantly if, in addition to shadowing, tilt modulation is taken into account. Hence, for grazing incidence and horizontal polarization, shadowing can be considered to be the dominant effect causing the differences between wave and image spectrum at high wavenumbers. Further we assume that effect of the variability of $\beta$ is small enough and can be neglected. Hence we consider $\beta=$ constant $=1.2$.

\section{Image inversion}

Using the empirical MTF derived above, it is possible to invert the sea clutter time series to estimate the waves imaged by the radar. The proposed method can be described in the following steps.

1) The 3D Fourier decomposition of the sea clutter time series $g(\mathbf{r}, t)$ is computed and their image amplitude and phase spectra, $A_{g}(\mathbf{k}, \omega)$ and $\varphi_{g}(\mathbf{k}, \omega)$, respectively, are estimated.
TABLE 3. Mean values and variance of the $\beta$ exponent derived from numerical simulations.

\begin{tabular}{llcc}
\hline \hline Sea state & \multicolumn{1}{c}{ Modulation } & Mean & Variance \\
\hline \multirow{2}{*}{ Swell } & Shadowing & 1.22 & 0.0276 \\
\multirow{3}{*}{ Wind sea } & Shadowing + tilt & 1.27 & 0.0317 \\
& Shadowing & 1.19 & 0.0057 \\
& Shadowing + tilt & 1.22 & 0.0078 \\
\hline
\end{tabular}

2) The high-pass filter of the spectra, $A_{g}(\mathbf{k}, \omega)$ and $\varphi_{g}(\mathbf{k}$, $\omega$ ), eliminates long-range dependence modulation effects, as the radar intensity power decay law with the range. Here an angular cutoff frequency of $\omega_{\text {cut }}$ $=0.188 \mathrm{rad} \mathrm{s}^{-1}\left(f_{\text {cut }} \approx 0.03 \mathrm{~Hz}\right)$ is used. Note that there are modern marine radars that already correct the range dependence (STC correction). Using this kind of modified radar data is not recommended because the modification has an unknown influence on the calibration.

3) The passband filter of the spectra extracts the waverelated components of $A_{g}(\mathbf{k}, \omega)$ and $\varphi_{g}(\mathbf{k}, \omega)$. Therefore, the relative motion between the radar and the wave field is determined first (see section 2). The filter band width is given by the angular frequency

(a) Swell simulation

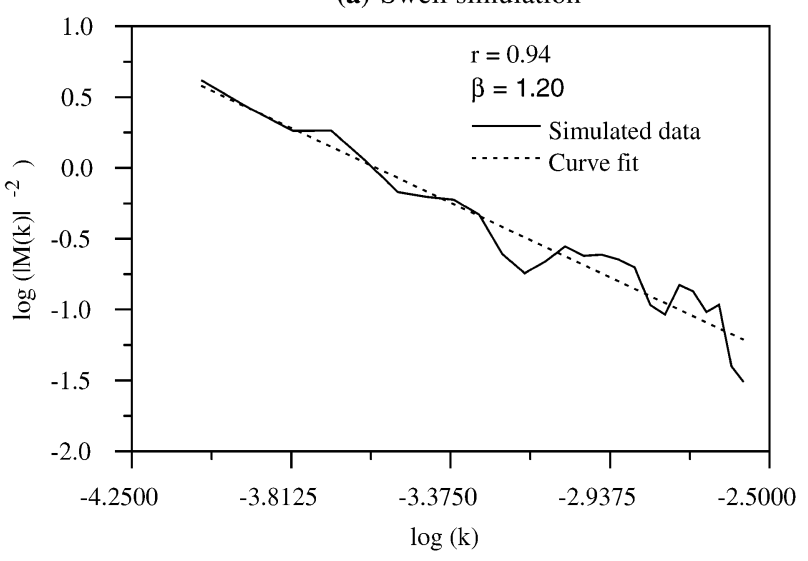

(b) Wind sea simulation

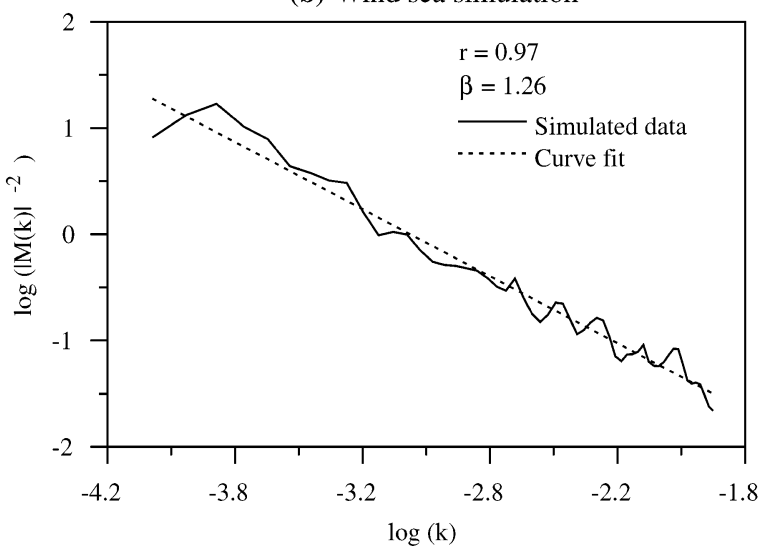

FIG. 5. Modulation transfer function exponent $\beta$ derived from (a) a swell simulation and (b) for a wind-sea simulation. 


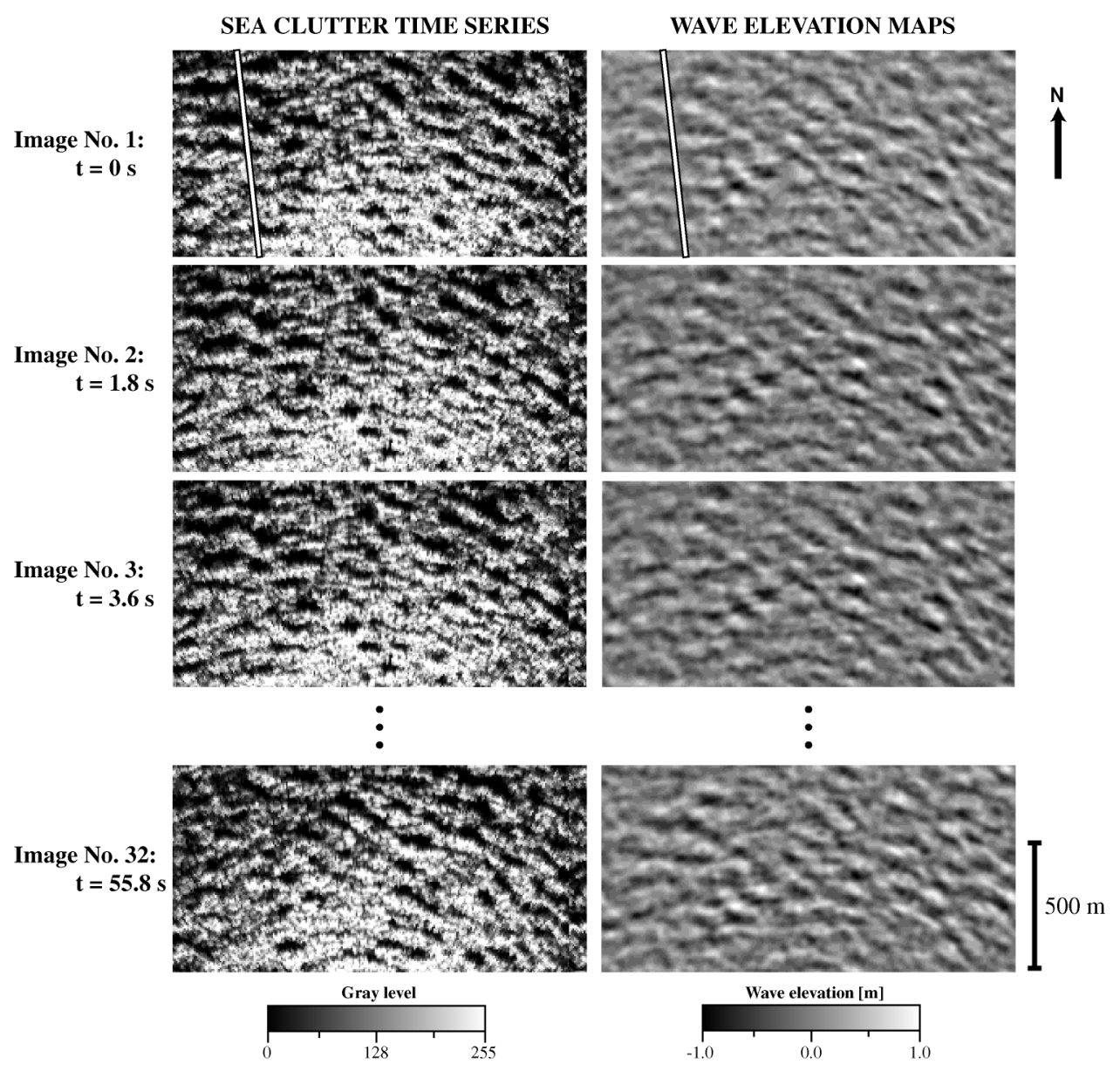

FIG. 6. (left) Sea clutter images and (right) corresponding wave elevation maps. The grayscales indicate the strength of the backscatter (left: black, no radar return; white, maximum radar return) and the surface elevation (right: black, minimum elevation: white, maximum elevation), respectively. In the upper two images, two lines indicate the position and orientation a transect depicted in Fig. 7.

resolution $\Delta \omega$. The corresponding angular cutoff frequencies, $\omega_{\text {cut } 1}$ and $\omega_{\text {cut } 2}$, are functions of the dispersion relation: $\omega_{\text {cut } 1,2}(\mathbf{k})=\varpi(\mathbf{k}) \pm \Delta \omega$.

4) The wave amplitudes $A_{\tilde{\eta}}(\mathbf{k}, \omega)$ are determined by applying the inverse MTF to the filtered amplitude spectrum $A_{g}(\mathbf{k}, \omega)$, where $\beta=1.2$ is used. Note that the nonlinear image mechanisms also affect the phases. This is mainly important for low incidence angles (in that case near range) when tilt is the dominant modulation. As we work at far range and grazing incidence, we assumed that the nonlinear effects on the phases are negligible.

5) The sea surface elevation $\tilde{\eta}(\mathbf{r}, t)$ is reconstructed by inverse DFT using the filtered wave amplitude $A_{\tilde{\eta}}(\mathbf{k}$, $\omega)$ and phase spectra $\varphi_{g}(\mathbf{k})$. Note that, because the wave amplitudes are not calibrated, $\tilde{\eta}(\mathbf{r}, t)$ gives nonscaled values of the sea surface elevation.

6) Here $\tilde{\eta}(\mathbf{r}, t)$ is scaled by using the relation $\sigma_{\eta}=$ $H_{s} / 4$, where $\sigma_{\eta}$ is the standard deviation of the wave elevation, and $H_{s}$ is derived from the SNR radar measurement (see section 2). Hence the calibrated estimation of the sea surface elevation $\eta(\mathbf{r}, t)$ is given by

$$
\eta(\mathbf{r}, t)=c \tilde{\eta}(\mathbf{r}, t), \quad \text { with } c=\frac{H_{s}}{4 \sigma_{\tilde{\eta}}},
$$

where $\sigma_{\tilde{\eta}}$ is the standard deviation of $\tilde{\eta}(\mathbf{r}, t)$.

Figure 6 shows an example of a temporal sequence of wave elevation maps synthesized by using the scheme described above. The sea clutter images were obtained at 2000 UTC 10 February 2000 by the permanent WaMoS II station Cabo de Peñas, which is located in the northern coast of Spain. The mean water depth is about $h=45 \mathrm{~m}$. The distance between the radar antenna and the shown area is about $2000 \mathrm{~m}$. The antenna is located at $100 \mathrm{~m}$ above the mean sea level. The sampling time of the image sequence (i.e., the antenna rotation period) is $1.8 \mathrm{~s}$. The WaMoS II determined for this dataset a significant wave height of $1.3 \mathrm{~m}$, with a peak period of $8.6 \mathrm{~s}$ and a peak wavelength of $115 \mathrm{~m}$. Two wave systems can be found with propagation directions 


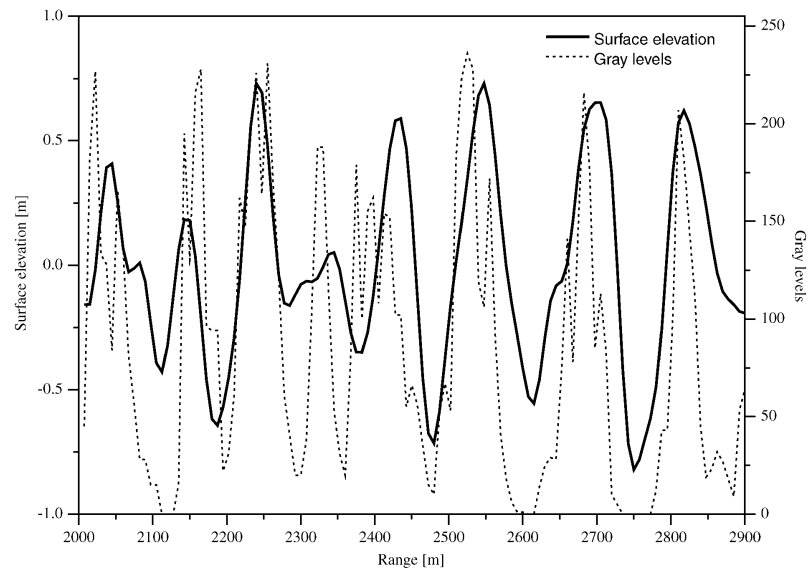

FIG. 7. Overlaid plot of the sea clutter transect (dashed line) and the corresponding wave elevation transect (solid line) as depicted in Fig. 6.

of $182^{\circ}$ and $209^{\circ}$. In the left row of the figure the examples of sea clutter images for different time steps are shown. The gray levels are related to the radar backscatter intensity, where black indicates no radar return. In the right row of the figure the corresponding wave elevation maps are shown, where the gray level indicates the surface elevation. In the upper images a line indicates a transect in range direction for which the gray level intensity $g$ and the wave elevation $\eta$ are depicted in Fig. 7. As expected the highest radar returns are found near the wave crests. Note that the radar antenna is on the left side. Behind the wave crests minimum radar return due to shadowing is visible. The transect of the sea surface elevation allows the investigation of single wave properties, such as wave height, steepness, etc. Following the zero-up crossing definition for time series, we identified the fifth wave (at the distance of about $500 \mathrm{~m}$ ) as the maximum wave with a height of $1.45 \mathrm{~m}$ and steepness of 0.011 . Figure 8 illustrates the comparison of the frequency spectra derived, respectively, from the estimated wave elevation map (thick line) and a buoy record (thin line). Note that both spectra present a good agreement.

\section{Estimation of wave height probability distribution function}

From the wave elevation maps $\eta(\mathbf{r}, t)$, it is possible to derive the probability density function (PDF) of wave heights. For buoy wave records (e.g., wave elevation time series), wave heights can be determined from the zero-up crossing method, and therefore the wave height PDF can be estimated. A multidimensional generalization of the zero-up crossing method is difficult to carry out, mainly when the wave fields are composed of several single wave systems with different propagation directions. For those cases when there is not an evident dominant wave propagation direction, it is not obvious

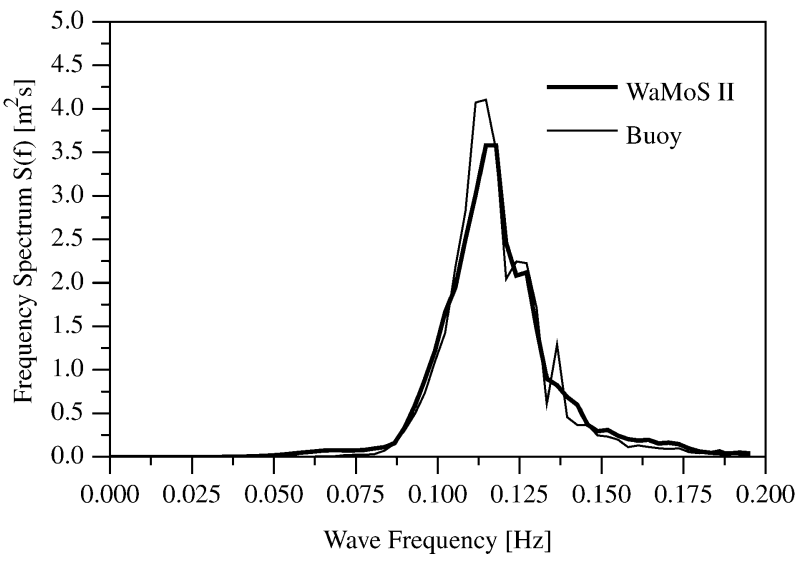

FIG. 8. Comparison of the frequency spectra derived from the buoy record and the wave elevation map shown in Fig. 6 .

what transect should be chosen to determine the wave heights.

For the unidimensional case, an alternative solution to determine the wave height PDF is based on the use of the wave envelope (Rice 1958; Longuet-Higgins 1986) and its determination by the Hilbert transform. Therefore, the wave elevation $\eta(t)$ is factorized as $\eta(t)$ $=a(t) \cos \phi(t)$, where $a(t)$ is the instantaneous amplitude and $\phi(t)$ is the instantaneous phase. Those are given, respectively, by

$$
a(t)=\sqrt{\eta^{2}(t)+\hat{\eta}^{2}(t)} ; \quad \phi(t)=\tan ^{-1}\left[\frac{\hat{\eta}(t)}{\eta(t)}\right],
$$

where $\hat{\eta}(t)$ is the Hilbert transform of $\eta(t)$.

When $\eta$ is a Gaussian process, the amplitude $a$ is Rayleigh distributed. In addition, when $\eta$ as a narrowbanded process, it is possible to determine the wave height probability assuming that the wave height is twice the amplitude. Hence, by transforming the random variable $a$ into $H=2 a$ in the PDF of wave amplitudes, the wave height PDF is a Rayleigh-distributed variable as well (Longuet-Higgins 1986; Ochi 1998; Goda 2000).

To generalize this idea to three dimensions, it is necessary to estimate the local and instantaneous amplitude $a(\mathbf{r}, t)$. For higher dimensions, there is not a unique definition of the Hilbert transform. This work uses the generalization proposed by Stark (1971). Hence, the socalled 3D total Hilbert transform $\hat{\eta}(\mathbf{r}, t)$ is given by

$$
\hat{\eta}(\mathbf{r}, t)=\frac{1}{\pi^{3}} \mathcal{P} \int_{\mathbb{R}^{3}} \frac{\eta(\boldsymbol{\lambda}, \tau)}{\left(x-\lambda_{x}\right)\left(y-\lambda_{y}\right)(t-\tau)} d \lambda_{x} d \lambda_{y} d \tau,
$$

where $P$ stands for the Cauchy's principal value of the integral, and $\boldsymbol{\lambda}=\left(\lambda_{x}, \lambda_{y}\right)$ is defined in the same spatial domain as $\mathbf{r}$. Therefore, the wave elevation image time series $\eta(\mathbf{r}, t)$ is factorized as $\eta(\mathbf{r}, t)=a(\mathbf{r}, t)$ $\cos \phi(\mathbf{r}, t)$, where $a(\mathbf{r}, t)$ is the local and instantaneous amplitude and $\phi(\mathbf{r}, t)$ the local and instantaneous phase. 

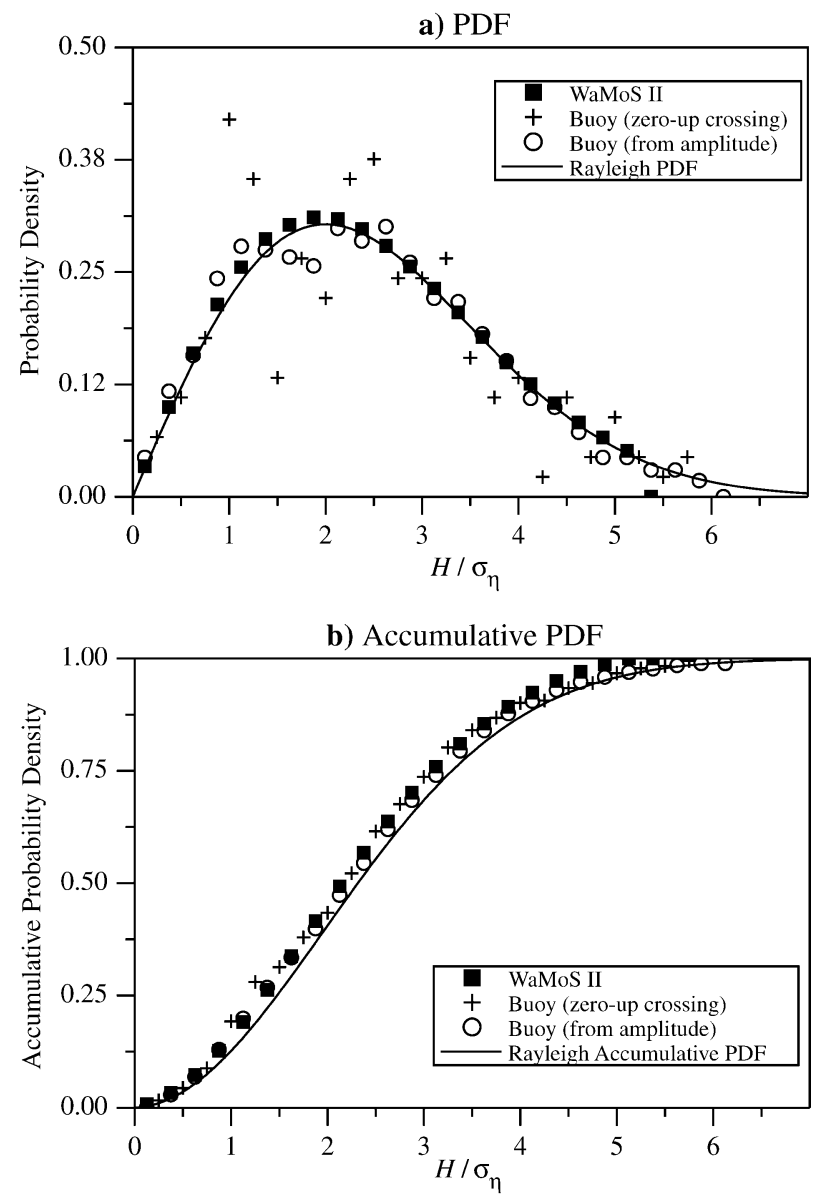

FIG. 9. (a) Rayleigh PDF (solid line) and histograms wave height derived from zero-up crossing analysis of a buoy record (crosses), wave height computed from the envelope estimated by the Hilbert transform of the buoy heave time series (circles), and wave height derived from the envelope estimated by the 3D total Hilbert transform of the inverted WaMoS II image time series (black squares). (b) Corresponding accumulative histograms and Rayleigh PDF. The wave heights $H$ have been normalized dividing by the std dev of the wave elevations $\sigma_{\eta}$. The data correspond to the wave elevation map time series shown in Fig. 6.

The local and instantaneous amplitude $a(\mathbf{r}, t)$ permits us to obtain the amplitude histogram. Assuming again the narrowband hypothesis, the wave height PDF can be estimated from the amplitude histogram by changing the random variable $a$ into $H=2 a$.

Figure 9 shows the wave height histograms (symbols) and the Rayleigh probability density function (solid line). The black squares indicate the results from the wave elevation maps by using the total Hilbert transform. The crosses indicate the results for the buoy records by means of zero-up crossing analysis. The circles correspond to the results for the buoy record using the 1D Hilbert transform. The results for the wave elevation maps show a good correlation with the theoretical Rayleigh distribution.

\section{Summary and conclusions}

The proposed inversion method allows us to estimate sea surface elevation maps from sea clutter images acquired by marine radar. This method represents an extension of an existing technique to estimate wave spectra from temporal sequences of marine radar images. It is based on the linear wave theory and assumes the wave field homogeneous in space and stationary in time.

To develop this inversion technique, an investigation was carried out to determine empirically the modulation transfer function (MTF) for marine radar measurements (grazing incidence and horizontal polarization). This MTF was obtained by using marine radar measurements and reference buoy. The MTF was fitted to a power decay law of wavenumbers, where the MTF exponent $\beta$ presents a mean value of 1.2 . To obtain additional information about the main imaging mechanisms responsible of the radar imagery, various numerical simulations of swell and wind sea were carried out. For each sea state simulation, the respective radar image was determined assuming shadowing only and shadowing plus tilt modulation. For all the cases, the same type of power decay law was found, with similar fit values of $\beta$. In addition, the simulation results showed that shadowing had a major influence and tilt modulation only a minor influence on MTF. These results allows to consider $\beta=$ constant $=1.2$.

The inversion scheme was applied to another dataset, which was not used for the previous investigation of MTF and its $\beta$ exponent. The comparison of the wave spectrum obtained from a buoy record and from the estimated wave elevation maps show good agreement, which supports the $\beta=1.2$ assumption.

The overlaid plot of the radar backscatter and the corresponding estimation of the wave elevation map along a transect demonstrated the capability of the method to remove the shadowing. It also demonstrated the possibility to investigate single wave properties is space.

For an additional validation of the inversion method, the wave height probability density function was determined from the wave elevation maps and a reference buoy. As there is no existing standard technique to determine wave height from spatial data, a general solution based on the use of the total 3D Hilbert transform was presented. The total Hilbert transform allows an estimate of the local and instantaneous wave envelope and hence the wave height probability density function, assuming the wave field as a narrowbanded process. To compare the results with buoy data the corresponding wave elevation time series were analyzed by means of the standard zero-up crossing method, as well as by the instantaneous wave envelope computed from the 1D Hilbert transform. The results also show a good agreement with the theoretical Rayleigh distribution.

As a final remark, it should be mentioned that the inversion method described in this paper is based on an empirical estimation of the MTF, which has been derived 
from normalized spectra. Hence the amplitudes of the wave spectrum and wave elevation maps resulting from the inversion scheme are estimated using the SNR calibration of the radar. For future investigations, an expanded the knowledge of the radar imaging mechanisms for grazing incidence and horizontal polarization will be important in order to obtain a more sophisticated MFT including both phase and amplitude spectra. This would also improve the proposed inversion scheme.

Acknowledgments. This work has been carried out in the frame of the European research project Maxwave (EU Contract EVK:3-2000-00544). In addition, the authors wish to thank the Spanish Port and Harbor Administration (Puertos del Estado) for the provision of the WaMoS II and buoy data, which were collected as a task of the European research project EuroROSE (EU Contract CT98-0168), and the Spanish Marine Safety and Rescue Society (SASEMAR) and the crew of the rescue vessel Golfo de Vizcaya.

\section{REFERENCES}

Alpers, W., and K. Hasselmann, 1982: Spectral signal to clutter and thermal noise properties of ocean wave imaging synthetic aperture radars. Int. J. Remote Sens., 3, 423-446.

Buckley, J. R., and J. Aler, 1997: Estimation of ocean surface wave height from grazing incidence microwave backscatter. Proc. Int. Geoscience and Remote Sensing Symp., Singapore, China, 10151017.

_ , and _ 1998: Enhancements in the determination of ocean surface wave height from grazing incidence microwave backscatter. IGARSS 98 Proc., Seattle, WA.

Dittmer, J., 1995: Use of marine radars for real time wave field survey and speeding up the transmission process. Proc. WMO/IOC Workshop on Operational Ocean Monitoring Using Surface Based Radars.

Goda, Y., 2000: Random Seas and Design of Maritime Structures. World Scientific, 443 pp.

Henschel, M. D., J. R. Buckley, and F. W. Dobson, 1995: Estimates of wave height from low incidence angle sea clutter. Proc. Fourth Int. Workshop on Wave Hindcasting and Forecasting, Banff, AB, Canada, Federal Panel on Energy R\&D, E-3.

Hessner, K., K. Reichert, and J. Dittmer, 1999: Coastal application of a wave monitoring system based on nautical radar. Proc. IGARSS, 99, Hamburg, Germany.

, J. C. Nieto Borge, and H. Günther, 2001: Evaluation of WaMoS II wave data. Proc. Fourth Int. Symp. on Ocean Wave Measurement and Analysis, San Francisco, CA, ASCE, 221-230.

Huang, N., 1981: A unified two-parameter wave spectral model for a general sea state. J. Fluid Mech., 112, 203-224.

Lee, P. H. Y., and Coauthors, 1995: X-Band microwave backscattering from ocean waves. J. Geophys. Res., 100, 2591-2611.

Longuet-Higgins, M. S., 1986: Wave group statistics. Oceanic Whitecaps, E. Monahan and G. Mac Niocaill, Eds., D. Reidel, 15-35.
Mitsuyasu, H., F. Tasai, T. Suhara, S. Mizuno, M. Ohkusu, T. Honda, and K. Rikiishi, 1980: Observation of the power spectrum of ocean waves using a coverleaf buoy. J. Phys. Oceanogr., 10, 286-296.

Nieto Borge, J. C., 1997: Análisis de campos de oleaje mediante radar de navegación en Banda X. Ph.D. thesis, Universidad de Alcalá de Henares, 320 pp.

_ - 1998: Significant wave height estimation from nautical radar data sets. GKSS Research Center Geesthacht Rep. 98/E/28, Geesthacht, Germany, 34 pp.

—_ and C. Guedes Soares, 2000: Analysis of directional wave fields using X-Band navigation radar. Coastal Eng., 40, 375-391.

— K. Kessner, and K. Reichert, 1999a: Estimation of the significant wave height with X-Band nautical radars. Proc. 18th Int. Conf. on Offshore Mechanics and Arctic Engineering, St. Johns, NF, Canada, ASME, CD-ROM OMAE99/OSU3063.

—, K. Reichert, and J. Dittmer, 1999b: Use of nautical radar as a wave monitoring instrument. Coastal Eng., 37, 331-342.

Ochi, M. K., 1998: Ocean Waves: The Stochastic Approach. Cambridge University Press, 319 pp.

Plant, W. H., 1988: The modulation transfer function: Concept and applications. Radar Scattering from Modulated Wind Waves, G. J. Komen and W. A. Oost, Eds., Kluwer Academic, 155-172.

Plant, W. J., and W. C. Keller, 1990: Evidence of Bragg scattering in microwave Doppler spectra of sea return. J. Geophys. Res., 95, 16 299-16 310 .

_- and L. M. Zurk, 1997: Dominant wave directions and significant wave heights from SAR imagery of the ocean. J. Geophys. Res., 102, 3473-3482.

Rice, S. O., 1958: Distribution of the duration of the random noise. Bell Syst. Tech. J., 37, 581-635.

Seemann, J., 1997: Interpretation der Struktur des Wellenzahl-Frequenzspektrums von Radar-Bildsequenzen. Ph.D. thesis, Universität Hamburg, 257 pp.

_ - F. Ziemer, and C. M. Senet, 1997: A method for computing calibrated ocean wave spectra from measurements with a nautical X-Band radar. Proc. Conf. on 500 years of Ocean Explorations, Halifax, NS, Canada, IEEE, 1148-1154.

Senet, C. M., J. Seemann, and F. Ziemer, 2001: The near-surface current velocity determined from image sequences of the sea surface. IEEE Trans. Geosci. Remote Sens., 39, 492-505.

Sorensen, R., 1993: Basic Wave Mechanics for Coastal and Ocean Engineers. Wiley \& Sons, 304 pp.

Stark, H., 1971: An extension of the Hilbert Transform Product Theorem. Proc. IEEE, 59, 1359-1360.

Wenzel, L. B., 1990: Electromagnetic scattering from the sea at low grazing angles. Suface Waves and Fluxes, G. L. Geernaert and W. J. Plant, Eds., Kluwer Academic, 41-108.

Young, I. R., W. Rosenthal, and F. Ziemer, 1985: Three-dimensional analysis of marine radar images for the determination of ocean wave directionality and surface currents. J. Geophys. Res., 90, 1049-1059.

Ziemer, F., and W. Rosenthal, 1991: Directional spectra from shipboard navigation radar during LEWEX. Directional Ocean Wave Spectra: Measuring, Modeling, Predicting, and Applying, R. C. Beal, Ed., The Johns Hopkins University Press, 125-127.

- and H. Günther, 1994: A system to monitor ocean wave fields. Proc. Second Int. Conf. on Air-Sea Interaction and Meteorology and Oceanography of the Coastal Zone, Lisbon, Portugal, Amer. Meteor. Soc., 18-19. 
Copyright of Journal of Atmospheric \& Oceanic Technology is the property of American Meteorological Society and its content may not be copied or emailed to multiple sites or posted to a listserv without the copyright holder's express written permission. However, users may print, download, or email articles for individual use. 